\title{
Compact Microstrip Antenna with Pattern-Reconfigurable Characteristic
}

\author{
Wei-Dong MA, Guang-Ming WANG, Ya-Wei WANG, Bin-Feng ZONG \\ Air and Missile Defense College, Air Force Engineering University, Shaanxi Province 710051, China \\ maweidong607@126.com,wgming01@sina.com \\ Submitted May 23, 2016 / Accepted March 2, 2017
}

\begin{abstract}
In this paper, a compact microstrip antenna with radiation pattern reconfigurable characteristic employing MEMS switches is proposed and investigated. This novel antenna mainly includes four parts: a circular-shaped patch, six MEMS switches, six fan-shaped coupling cells and six fan-shaped radiation cells. Through controlling the states of MEMS switches, radiation pattern selectivity of the proposed antenna can be achieved. When switching operation states of the antenna from state 1 to state 12 sequentially, the main radiation beam direction in azimuth planes could rotate $30^{\circ}$ from $0^{\circ}$ to $330^{\circ}$ with each switch. Meanwhile, the radiation beam directions in elevation plane and the operation frequencies of the proposed antenna for different operation states are stable at about $35^{\circ}$ and $4 \mathrm{GHz}$, respectively. With the consideration of MEMS switches actual size, the antenna structure and dimensions were optimized and adjusted. The simulated and measured results were analyzed and compared, indicating this compact antenna can be widely used for multifunctional applications and modern wireless communication systems.
\end{abstract}

\section{Keywords}

Reconfigurable antenna, compact microstrip antenna, radiation pattern reconfigurability, MEMS switch

\section{Introduction}

Reconfigurable antenna (RA) for the function of changing its electrical parameters dynamically in respond to various environments has been studied extensively [1]. RA can satisfy the diverse requirements and strict standards of advanced wireless communication systems due to its numerous merits such as reducing size, expanding capacity, improving security and lessening interference [2], [3]. According to the alterations of electrical parameters, RAs can be mainly divided into four categories: frequency-reconfigurable antenna (FRA), polarization-reconfigurable antenna (PoRA), pattern-reconfigurable antenna (PaRA), and multiparameter-reconfigurable antenna (MRA) [4].

In recent years, the PaRA is attracting more and more attention as it is very useful for systems which suffer from multipath interference [5]. On account of its pattern selectivity, the PaRA is capable of enhancing system security, improving communication reliability and reducing energy loss to better the comprehensive performance of whole system. The textbook approach to antenna pattern reconfiguration is using phased-array antenna with fast response. However, the phased array antenna usually has a large volume and complex feed-network. Therefore, a lot of substantial research work has been carried out to PaRAs with electrical tuning. Electronic switch components, such as field effect transistors (FETs), micro-electromechanical systems (MEMSs), PIN diodes and varactors, are often used to reconfigure the radiation patterns of antennas [6-9].

Compared with traditional and classical switches, MEMS switch made on a common silicon substrate is tiny enough for integration. Due to its advantageous properties such as high isolation, good linearity, wide band and low loss, RF MEMS switches have been used in reconfigurable antennas for many years [10-12].

Designs resorted MEMS switch to achieve radiation pattern reconfigurability can be found in many literatures [13-15]. A reconfigurable microstrip antenna presented in [13] uses MEMS switches for pattern selectivity realization. The reconfigurable antenna provides three radiationpattern modes by controlling the states of four MEMS switches. In [14], the reconfigurable rectangular spiral antenna could radiate at different directions because that the four MEMS switches can switch the connection or disconnect of five discrete arms. However, each resonant state can only cover a narrow range. The antenna shown in [15] can realize the operation frequency, radiation pattern and polarization mode reconfiguration simultaneously by switching MEMS switches. And the impacts of bias circuit network and feed-network on the efficiency of antenna are important to the design and need to be taken into account. In these designs, MEMS switches are selected to reconfigure the radiation patterns of the antennas mainly because that they can provide superior performance than active switches such as PIN diodes, and be integrated in these designs conveniently.

In this paper, a compact microstrip antenna with radiation pattern reconfigurability using MEMS switches is proposed and investigated. Six MEMS switches are used to respectively connect or disconnect the fan-shaped coupling 
cells and fan-shaped radiations cells of the proposed antenna, changing the current distribution and achieving radiation pattern diversity. Twelve basic operation states (state 1 to state 12) are obtained for the proposed antenna. Sequentially switching operation states from state 1 to state 12 can rotate the main radiation beam by $30^{\circ}$ from $0^{\circ}$ to $330^{\circ}$ in azimuth plane, maintaining $35^{\circ}$ in elevation plane. Moreover, this novel PaRA can operate around $4 \mathrm{GHz}$ stably with all operation states, indicating that it is a good candidate for advanced communication systems of, for example, satellite $\mathrm{C}$ band $(3.7 \mathrm{GHz} \sim 4.2 \mathrm{GHz})$. Moreover, the proposed PaRA possesses of many merits such as low cost, compact structure and stable radiation performance.

\section{Antenna Design and Analysis}

\subsection{Antenna Configuration}

The top and side views of the proposed PaRA are illustrated in Fig. 1 detailedly. This novel PaRA designed on a FR4 substrate (dielectric constant $\varepsilon_{\mathrm{r}}=4.3$, loss tangent $\tan \delta=0.02$ and thickness $H=3 \mathrm{~mm}$ ), mainly consists of four parts. The first (red area) part is a circular-shaped patch located at the center of the proposed antenna for connecting a 50- $\Omega$ coaxial cable. The second (blue area) and third (yellow area) parts are six fan-shaped coupling cells and six fan-shaped radiation cells respectively, which distribute around the antenna center symmetrically. Six RF switches, the fourth part, are used to connect or disconnect the coupling cells and radiation cells, hence change the radiation structure. When the switch shifts to ON-state, the radiation cell connected to the corresponding coupling cell will be activated. Contrarily, the radiation cell will not work when the switch shifts to OFF-state. With controlling the states of switches, the proposed PaRA can operate at different states and achieve radiation pattern reconfigurable characteristic.

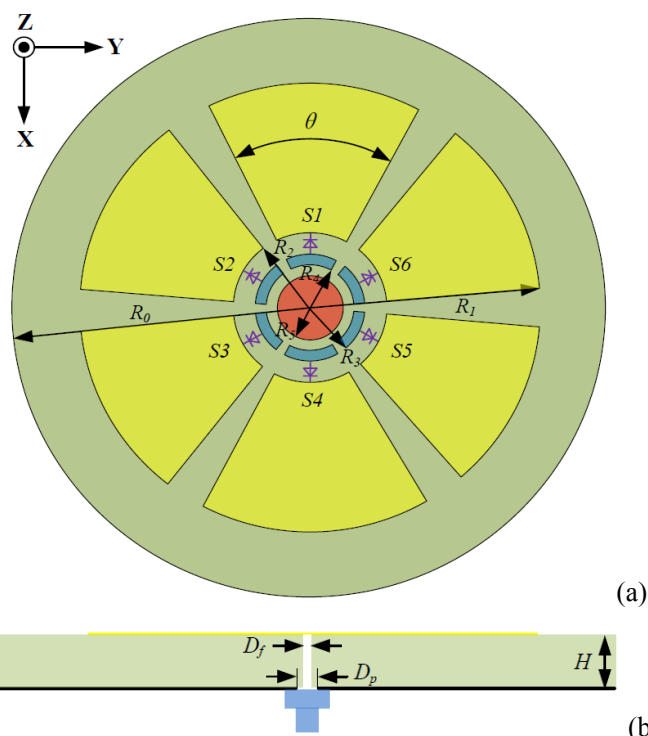

(b)

Fig. 1. Geometry of the proposed PaRA: (a) top, (b) side view.

\subsection{MEMS Switch and Bias Circuit}

Six Radant MEMS RMSW101 switches (S1 S6) with advantageous properties of small size, high isolation, low loss and high linearity, are selected as electrical components to reconfigure the radiation pattern of the proposed PaRA. For MEMS switch actuation, $90 \mathrm{~V}$ DC voltage between the source and gate is required, which will pull the free end of the cantilever into contact with the drain. The data book of the used type of MEMS switch shows that at the operation frequency $4 \mathrm{GHz}$, the insertion loss is about $0.26 \mathrm{~dB}$ in the $\mathrm{ON}$-state which brings in RF continuity. Also the isolation is $21 \mathrm{~dB}$ in the OFF-state which results in RF discontinuity.

The bias circuit network for MEMS switch actuation is designed according to the data book of MEMS switch. The source and gate of MEMS switch are respectively connected the pads in the bias line for DC actuation voltage loading. Moreover, the source and drain of the MEMS switch is required to connect to the DC ground plane. For circuit simplicity, the RF ground plane is used for the DC ground plane. And the DC continuity between antenna and ground can be achieved with stub line through via structure Besides, DC biasing lines are properly designed to reduce the effect of bias current to radiation characteristic and matching characteristic of antenna. $100 \mathrm{k} \Omega$ SMD (Surface Mounted Devices) resistances and $270 \mathrm{nH}$ SMD inductances are applied in biasing lines to reduce the loss as well as the influence on antenna performance from biasing lines. In order to avoid the radiators are blocked by biasing lines in measurement and performance degradation of the antenna, the biasing lines are brought from the ground by via structure to connect the DC source.

\subsection{Parameters Analysis}

The parameters of the antenna determine its performance. The exradius $R_{1}$ of fan-shaped radiation cell is a major influence upon the operation frequency of the proposed antenna. Figure 2 plots the simulated operation frequency versus $R_{1}$. It displays that the operation frequency gradually decreases from $4.27 \mathrm{GHz}$ to $3.76 \mathrm{GHz}$ as $R_{1}$ increases from $19 \mathrm{~mm}$ to $22 \mathrm{~mm}$ with step increment of $1 \mathrm{~mm} . R_{1}=20.6 \mathrm{~mm}$ closed to a half guided-wave wavelength is selected as the appropriate parameter for that the

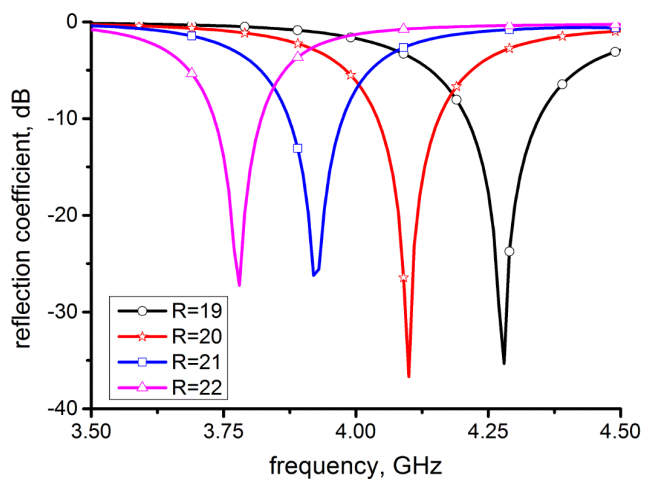

Fig. 2. Simulated operation frequency versus $R_{1}$. 


\begin{tabular}{|c|c|c|c|c|c|}
\hline Parameters & $R_{0}$ & $R_{1}$ & $R_{2}$ & $R_{3}$ & $R_{4}$ \\
\hline Dimensions & $62 \mathrm{~mm}$ & $20.5 \mathrm{~mm}$ & $5 \mathrm{~mm}$ & $4 \mathrm{~mm}$ & $3.5 \mathrm{~mm}$ \\
\hline Parameters & $R_{5}$ & $D_{\mathrm{f}}$ & $D_{\mathrm{p}}$ & $H$ & $\theta$ \\
\hline Dimensions & $3 \mathrm{~mm}$ & $1.4 \mathrm{~mm}$ & $4.1 \mathrm{~mm}$ & $3 \mathrm{~mm}$ & $50^{\circ}$ \\
\hline
\end{tabular}

Tab. 1. Dimensions of the proposed PaRA.

novel PaRA could operate at $4 \mathrm{GHz}$ exactly. Meanwhile, $\theta=50^{\circ}$ is obtained for good radiation and low-coupling. With consideration of the actual size of MEMS switch, the gap $\left(R_{2}-R_{3}\right)$ between fan-shaped coupling cell and fanshaped radiation cell is chosen as $1 \mathrm{~mm}$ for easy-fabrication. Furthermore, the radius $R_{5}$ of circular-shaped patch mainly affects the antenna's efficiency. And simulations show the efficiency arrive at its maximum when $R_{5}=3 \mathrm{~mm}$. In order to get good impedance match and high gain, the exradius $R_{3}=4 \mathrm{~mm}$ and inradius $R_{4}=3.5 \mathrm{~mm}$ of fanshaped coupling cell are acquired respectively. Lastly, the radius $R_{0}=62 \mathrm{~mm}$ and height $H=3 \mathrm{~mm}$ of the entire antenna is the optimized results for compact structure and low-profile ultimately.

The proposed PaRA is designed and analyzed with a popular EM simulated tool HFSS.14. The detailed antenna dimensions after optimization are listed in Tab. 1.

\subsection{Operation Principles}

With controlling the states of switches, different fanshaped radiation cell of the antenna can be fed selectively by the circular-patch through corresponding coupling cell. As a result, the surface current distribution of the antenna will be changed, realizing the deflection of the radiation direction of the antenna. Moreover, the diverse operation states of the proposed PaRA corresponding to different states of six MEMS switches are obtained. The amply simulated information of different operation states are summarized in Tab. 2. It shows that the proposed antenna has 12 basic operation states (one or two-adjacent ON-state MEMS switches), accordingly radiating at 12 different directions (from $0^{\circ}$ to $330^{\circ}$ with step increment of $30^{\circ}$ ) in azimuth planes and maintaining $35^{\circ}$ in elevation plane. Meanwhile, the operation frequencies of the proposed

\begin{tabular}{|c|c|c|c|c|c|c|c|c|c|}
\hline \multirow{2}{*}{$\begin{array}{c}\text { Operation } \\
\text { State }\end{array}$} & \multicolumn{6}{|c|}{ MEMS Switches* } & \multirow{2}{*}{$\begin{array}{c}\text { Resonance } \\
\text { Frequency } \\
(\mathrm{GHz})\end{array}$} & \multirow{2}{*}{$\begin{array}{c}\text { Beam } \\
\text { Direction } \\
\left({ }^{\circ}\right)\end{array}$} & \multirow{2}{*}{$\begin{array}{l}\text { Gain } \\
(\mathrm{dBi})\end{array}$} \\
\hline & S1 & $S 2$ & S3 & $S 4$ & S5 & S6 & & & \\
\hline state1 & 1 & 0 & 0 & 0 & 0 & 0 & $3.94 \sim 4.05$ & $\mathbf{0}$ & 5.16 \\
\hline state2 & 1 & 1 & 0 & 0 & 0 & 0 & $3.91 \sim 4.03$ & 30 & 5.75 \\
\hline state3 & 0 & 1 & 0 & 0 & 0 & 0 & $3.95 \sim 4.05$ & 60 & 5.16 \\
\hline state4 & 0 & 1 & 1 & 0 & 0 & 0 & $3.91 \sim 4.03$ & 90 & 5.75 \\
\hline state5 & 0 & 0 & 1 & 0 & 0 & 0 & $3.95 \sim 4.05$ & 120 & 5.16 \\
\hline state6 & 0 & 0 & 1 & 1 & 0 & 0 & $3.91 \sim 4.03$ & 150 & 5.75 \\
\hline state7 & 0 & 0 & 0 & 1 & 0 & 0 & $3.95 \sim 4.05$ & 180 & 5.16 \\
\hline state8 & 0 & 0 & 0 & 1 & 1 & 0 & $3.91 \sim 4.03$ & 210 & 5.75 \\
\hline state9 & 0 & 0 & 0 & 0 & 1 & 0 & $3.95 \sim 4.05$ & 240 & 5.16 \\
\hline state10 & 0 & 0 & 0 & 0 & 1 & 1 & $3.91 \sim 4.03$ & 270 & 5.75 \\
\hline state11 & 0 & 0 & 0 & 0 & 0 & 1 & $3.95 \sim 4.05$ & 300 & 5.16 \\
\hline state12 & 1 & 0 & 0 & 0 & 0 & 1 & $3.91 \sim 4.03$ & 330 & 5.75 \\
\hline
\end{tabular}

Tab. 2. The operation states of the antenna. antenna with different operation states all come around $4 \mathrm{GHz}$ and the 10-dB impedance bandwidths fluctuated at the range of $3.91 \mathrm{GHz}$ to $4.05 \mathrm{GHz}$ slightly. Similarly, the gains, as well as other performances of the proposed PaRA with different operation states are almost same with each other.

Note that other operation states (two-nonadjacent or more ON-state MEMS switches) of the proposed antenna almost have the similar radiation patterns with the 12 basic operation states (one or two-adjacent ON-state MEMS switches) in Tab. 2, respectively. However, the antenna performances of other operation states decrease observably though its operation frequencies change a little. After removing the redundant and invalid operation states with optimization and analysis, there are 12 basic operation states as shown in Tab. 2 are obtained. And in the following sections, these 12 basic operation states are mainly discussed, measured and summarized.

\section{Experimental Results of the PaRA}

A prototype shown in Fig. 3 was fabricated with commercial MEMS switches according to the optimized dimensions in Tab. 1. The experimental results of the proposed PaRA of 12 basic operation states are displayed as follows. Due to its symmetrical configuration, return losses of state 1 , state 3 , state 5 , state 7 , state 9 and state 11 are identical. Similarly, so do the return losses of state 2 , state 4 , state 6 , state 8 , state 10 and state 12 .

For briefness, Figure 4 shows the simulated and measured $\left|S_{11}\right|$ in $\mathrm{dB}$ of state 1 and state 2, respectively. Others can be obtained in the light of symmetry. It can be found that the simulated 10-dB impedance bandwidth $(3.95 \mathrm{GHz}$ $\sim 4.05 \mathrm{GHz}$ ) of state 1 is approximately the same with the bandwidth $(3.91 \mathrm{GHz} \sim 4.03 \mathrm{GHz})$ of state 2 . Moreover, the measured and simulated results agree very well.

\subsection{Radiation Pattern Reconfigurability}

When different MEMS switch shifting to ON-state, different fan-shaped radiation cell will be activated, making antenna radiation pattern diversity into realization. The simulated and measured 2-D radiation patterns of the proposed PaRA in azimuth plane for 12 basic operation states

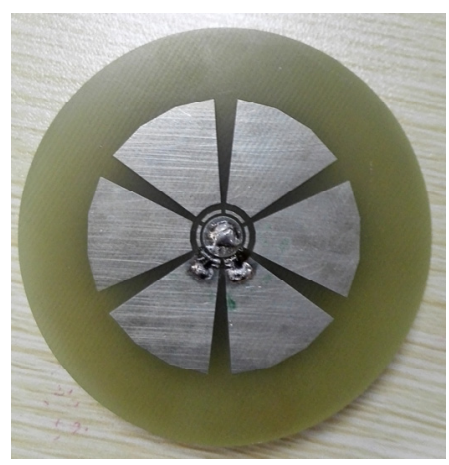

Fig. 3. Photograph of the fabricated antenna. 

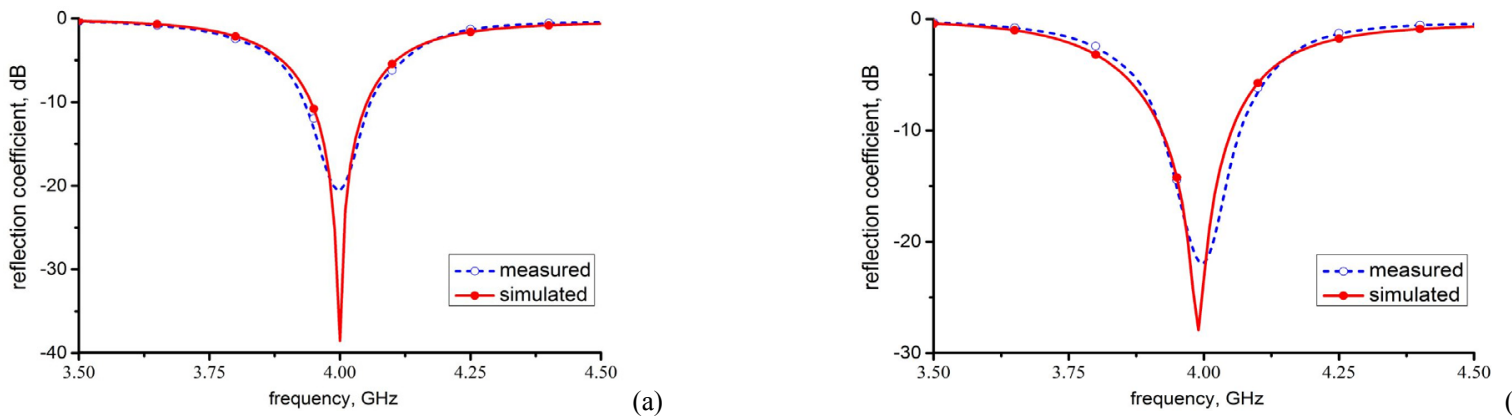

(b)

Fig. 4. Simulated and measured $\left|S_{11}\right|$ in $\mathrm{dB}$ of the proposed antenna (a) state 1, (b) state 2 .

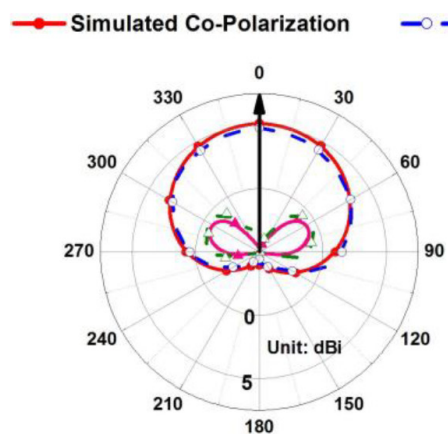

(a)

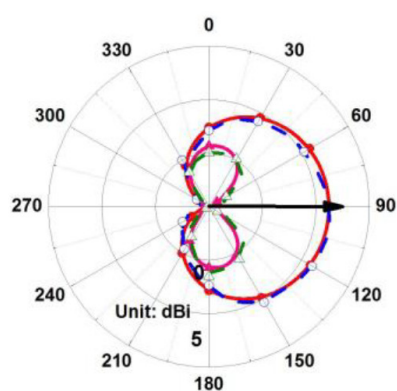

(d)

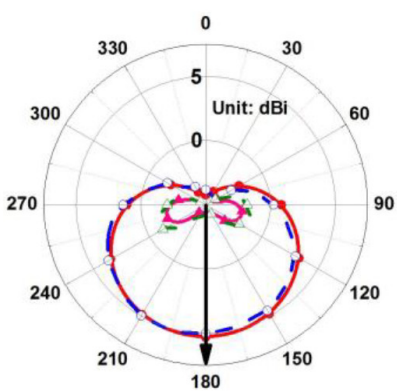

(g)

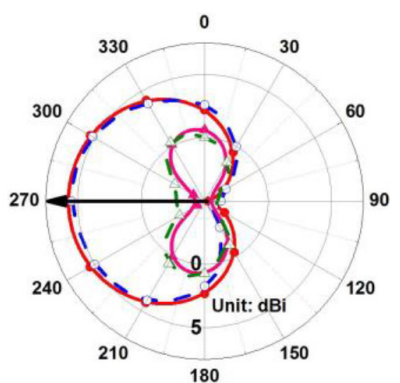

(j)

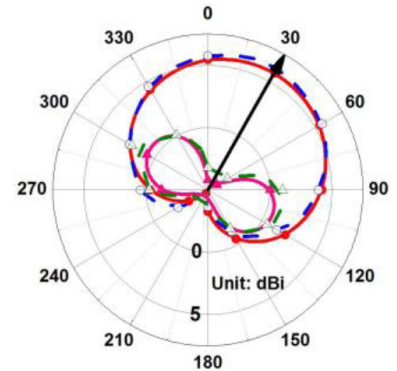

(b)

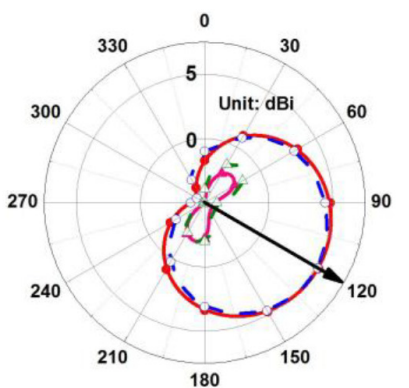

(e)

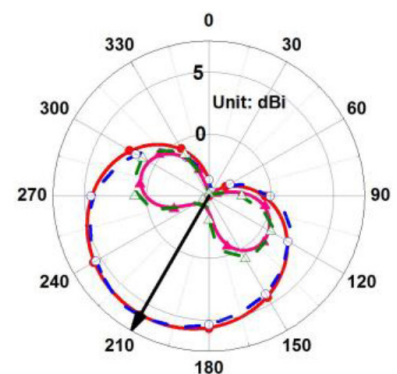

(h)

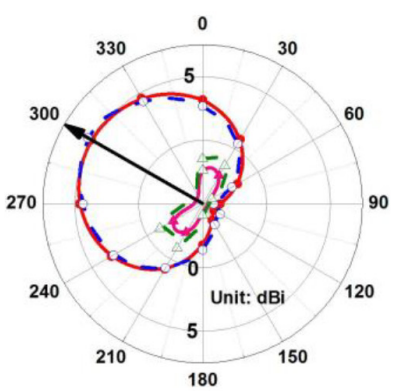

(k)

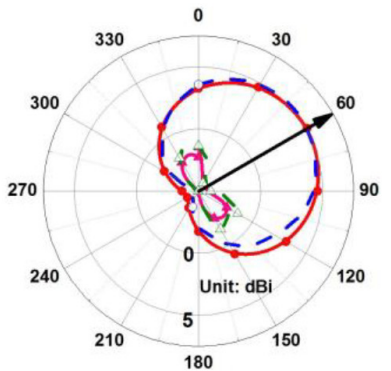

(c)

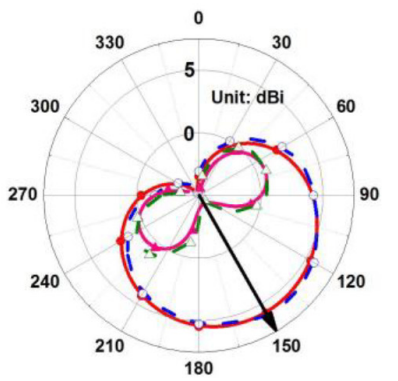

(f)

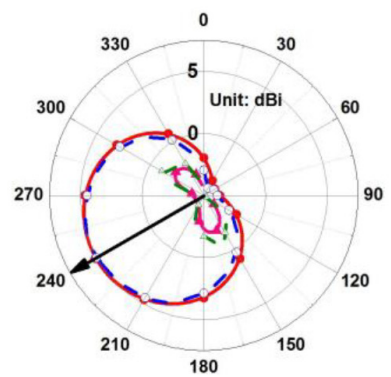

(i)

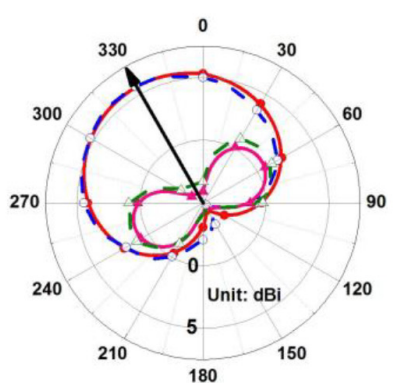

(1)

Fig. 5. Simulated and measured radiation patterns (azimuth plane) for different operation states : (a) state 1, (b) state 2, (c) state 3 , (d) state 4 , (e) state $5,(\mathrm{f})$ state $6,(\mathrm{~g})$ state $7,(\mathrm{~h})$ state 8 , (i) state $9,(\mathrm{j})$ state $10,(\mathrm{k})$ state $11,(\mathrm{l})$ state 12 . 
are shown in Fig. 5 in detail. From state 1 to state 12, the main beam direction of the proposed PaRA varies from $0^{\circ}$ to $330^{\circ}$ with step increment of $30^{\circ}$. It indicates that this novel design can cover the whole azimuth plane. It also manifests that each operation state provides corresponding main-beam direction and demonstrates the radiation pattern reconfigurable characteristic of the proposed PaRA.

\subsection{Antenna Performance}

For the 12 basic operating states of the proposed PaRA, its performances keep stable except the main beam direction in azimuth plane varies. Experimental results from measurement and simulation as follow demonstrate the performances of the proposed PaRA.

Figure 6 displays the measured 2-D radiation patterns of the proposed PaRA from state 1 to state 4 for briefness in elevation plane. For other operation states, similar results had been obtained. It can be observed from Fig. 6 that the radiation patterns in elevation are quite similar and the main beam directions all point at about $35^{\circ}$. Slight shifts appeared as a result of the fabricated errors and influences from MEMS switches and their biasing circuit network. The cross-polarizations of the radiation patterns in elevation plane are too small to be shown and so omitted.

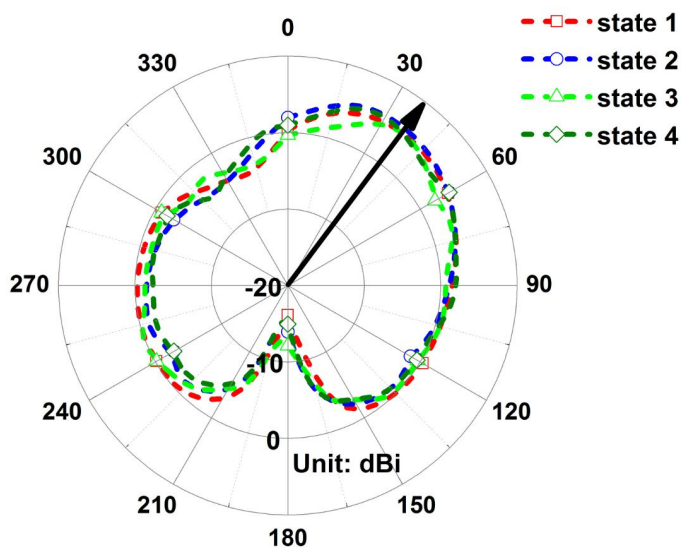

Fig. 6. Measured 2-D radiation patterns in elevation plane.

\begin{tabular}{|c|c|c|c|c|}
\hline $\begin{array}{c}\text { Operation } \\
\text { State }\end{array}$ & state1 & state2 & state3 & state4 \\
\hline Gain (dBi) & 5.02 & 5.60 & 5.11 & 5.54 \\
\hline Efficiency & $85.7 \%$ & $84.7 \%$ & $86.1 \%$ & $85.3 \%$ \\
\hline $\begin{array}{c}\text { Operation } \\
\text { State }\end{array}$ & state5 & state6 & state7 & state8 \\
\hline Gain (dBi) & 5.05 & 5.63 & 5.03 & 5.69 \\
\hline Efficiency & $85.9 \%$ & $86.6 \%$ & $85.3 \%$ & $87.1 \%$ \\
\hline $\begin{array}{c}\text { Operation } \\
\text { State }\end{array}$ & state9 & state10 & state11 & state12 \\
\hline Gain (dBi) & 5.14 & 5.61 & 5.09 & 5.55 \\
\hline Efficiency & $86.9 \%$ & $85.4 \%$ & $85.6 \%$ & $84.9 \%$ \\
\hline
\end{tabular}

Tab. 3. Measured gains and efficiencies of the proposed PaRA.
The measured realized gains and efficiencies of the proposed PaRA with 12 different operation states are summarized in Tab. 3. It can be discovered that the realized gains for different operating states are all more than $5 \mathrm{dBi}$ and efficiencies for different operating states are mostly up to $85 \%$. Moreover, the experiment results of measurement and simulation are compared in detail. The results not only showed a good agreement but also demonstrated the stable and good performances of the proposed PaRA.

\section{Conclusion}

A reconfigurable microstrip antenna with radiation pattern selectivity using MEMS switches is designed and investigated. The novel PaRA has compact structure with circle patch, radiation cells, coupling cells and MEMS switches. With controlling the states of MEMS switches, the proposed PaRA can operate at 12 basic different states and achieve radiation pattern reconfigurable characteristic. When the operation states of the proposed PaRA steer sequentially, the main radiation beam direction can rotate by $30^{\circ}$ from $0^{\circ}$ to $330^{\circ}$ in azimuth plane. Meanwhile, the main beam directions in elevation plane and the operation frequencies of the PaRA with different states undulate slightly at $35^{\circ}$ and $4 \mathrm{GHz}$, respectively. Moreover, the realized gains, efficiencies as well as other performances of the PaRA are all very nice and stable. Besides, the novel PaRA possesses of many merits, such as low cost, compact structure and stable radiation performance, which indicate that it is well-suitable for multifunctional applications and modern communication systems.

\section{Acknowledgements}

This work is supported by the National Natural Science Foundation of China under Grant Nos. 61372034.

\section{References}

[1] HAUPT, R. L., LANAGAN, M. Reconfigurable antennas. IEEE Antennas and Propagation Magazine, 2013, vol. 55, no. 1, p. 4961. DOI: 10.1109/MAP.2013.6474484

[2] COSTANTINE, J., TAWK, Y., BARBIN, S. E., CHRISTODOULOU, C. G. Reconfigurable antennas: Design and applications. Proceedings of IEEE, 2015, vol. 103, no. 9, p. 425 to 437. DOI: 10.1109/JPROC.2015.2396000

[3] CHENG, Y.-J. Substrate integrated waveguide frequency-agile slot antenna and its multibeam application. Progress In Electromagnetics Research, PIER, 2012, vol. 130, p. 153-168. DOI: 10.2528/PIER12061602

[4] RODRIGO, D., CETINER, B. A., JOFRE, L. Frequency, radiation pattern and polarization reconfigurable antenna using a parasitic pixel layer. IEEE Transaction on Antennas and Propagation, 2014, vol. 62, no. 1, p. 3422-3427. DOI: 10.1109/TAP.2014.2314464 
[5] NGUYEN V.-A., JEONG. M.-H., DAO. M.-T, PARK. S.-O. Fourport beam reconfigurable antenna array for pattern diversity systems. IET Microwaves, Antennas and Propagation, 2015, vol. 6, no. 10 , p. 1179-1186. DOI: 10.1049/iet-map.2011.0606

[6] XIAO-LIN YANG, JIAN-CHENG LIN, GANG CHEN, FANGLING KONG. Frequency reconfigurable antenna for wireless communications using GaAs FET switches. IEEE Antennas and Wireless propagation Letters, 2015, vol. 14, p. 807-810. DOI: 10.1109/LAWP.2014.2380436

[7] KAGAN TOPALli, CIVI, O. A., DEMIR, S., et al. Dualfrequency reconfigurable slot dipole array with a CPW based feed network using RF MEMS technology for X- and Ka- band applications. In IEEE Antennas and Propagation Society International Symposium. Honolulu (HI, USA), 2007, p. 825-828. DOI: 10.1109/APS.2007.4395621

[8] LIN, X., YANG, X., KONG, F. A frequency monopole antenna with switchable stubbed ground structure. Radioengineering, 2015, vol. 24 , no. 2 , p. 449-454. DOI: $10.13164 /$ re.2015.0449

[9] BEHEDAD, N., SARABANDI, K. A varactor-tuned dual-band slot antenna. IEEE Transaction on Antennas and Propagation, 2006, vol. 54, no. 2, p. 401-408. DOI: 10.1109/TAP.2005.863373

[10] YASHCHYSHYN, Y. Reconfigurable antennas by RF switches technology. In 5th International Conference on Perspective Technology and Methods in MEMS Design (MEMSTECH 2009). Polyana-Svalyava (Ukraine), 2009, p. 155-157.

[11] ZOHUR, A., MOPIDEVI, H., RODRIGO, D., et al. RF MEMS reconfigurable two-band antenna. IEEE Antennas of Wireless Propagation Letters, 2013, vol. 12, p. 73-75. DOI: 10.1109/LAWP.2013.2238882

[12] GRAU, A., ROMEU, J., LEE, M.-J., et al. A dual-linearlypolarized MEMS-reconfigurable antenna for narrowband MIMO communication systems. IEEE Transaction on Antennas and Propagation, 2010, vol. 58, no. 1, p. 4-17. DOI: 10.1109/TAP.2009.2036197

[13] ZHANG, S., HUFF, G. H., FENG, J., et al. A pattern reconfigurable micro-strip parasitic array. IEEE Transaction on Antennas and Propagation, 2004, vol. 52, no. 10, p. 2773-2776. DOI: $10.1109 /$ TAP.2004.834372

[14] JUNG, C. W., LEE, M., LI, G. P., et al. Reconfigurable scan-beam single-arm spiral antenna integrated with RF-MEMS switches. IEEE Transaction on Antennas and Propagation, 2006, vol. 54, no. 2 , p. 455-463. DOI: 10.1109/TAP.2005.863407

[15] GRAU BESOLI, A., DE FLAVIIS, F. A multifunctional reconfigurable pixeled antenna using MEMS technology on printed circuit board. IEEE Transaction on Antennas and Propagation, 2011, vol. 59, no. 12, p. 4413-4424. DOI: 10.1109/TAP.2011.2165470

\section{About the Authors ...}

Wei-Dong MA was born in Shaanxi province, People's Republic of China. He received his B.S. from the China University of Geoscience, Wuhan, China, in 2012, and M.S degrees from the Air Force Engineering University, Xi'an, China, in 2014. His research interests include smart antenna, reconfigurable antenna, composite right/lefthanded (CRLH) transmission lines, and metamaterial-based antennas.

Guang-Ming WANG was born in Anhui province, People's Republic of China. He received his B.S. and M.S. degrees from the Missile Institute, Air Force Engineering University, Xi'an, China, in 1982 and 1990, respectively, and his Ph.D. degree from the Electronic Science and Technology University, Chengdu, China, in 1994. Then, he joined the Air Force Engineering University as a professor in 2000 and is now the head of the Microwave Laboratory Center in it. His current interest includes microwave circuits, antenna and propagation, and also the new structures include EBG, PBG, smart antenna, metamaterials and fractals, etc.

Ya-Wei WANG was born in Anhui province, People's Republic of China. He received his B.S. and M.S. degrees from the Air Force Engineering University, Xi'an, China, in 2008 and 2011, respectively. His research interests are mainly spiral antennas, metamaterial-based antennas, etc.

Bin-Feng ZONG was born in Jiangsu province, People's Republic of China. He received his B.S. and M.S. degrees from the Air Force Engineering University, Xi'an, China, in 2010 and 2012, respectively. His research interests include microstrip resonators, composite right/left-handed (CRLH) transmission lines and metamaterial-based antennas, etc. 